\title{
Moment transport equations for non-Gaussianity
}

\author{
David J. Mulryne ${ }^{1}$, David Seery ${ }^{1}$ and Daniel Wesley ${ }^{1,2}$ \\ ${ }^{1}$ Department of Applied Mathematics and Theoretical Physics \\ Wilberforce Road, Cambridge, CB3 0WA, United Kingdom \\ ${ }^{2}$ Center for Particle Cosmology \\ David Rittenhouse Laboratory, University of Pennsylvania \\ 209 South 33rd Street, Philadelphia, PA 19104 USA
}

E-mail: djm63@cam.ac.uk, djs61@cam.ac.uk, dwes@sas.upenn.edu

Submitted to: JCAP

PACS numbers: 98.80.-k, 98.80.Cq, 11.10.Hi

\begin{abstract}
We present a novel method for calculating the primordial non-Gaussianity produced by super-horizon evolution during inflation. Our method evolves the distribution of coarse-grained inflationary field values using a transport equation. We present simple evolution equations for the moments of this distribution, such as the variance and skewness. This method possesses some advantages over existing techniques. Among them, it cleanly separates multiple sources of primordial nonGaussianity, and is computationally efficient when compared with popular alternatives, such as the $\delta N$ framework. We adduce numerical calculations demonstrating that our new method offers good agreement with those already in the literature. We focus on two fields and the $f_{\mathrm{NL}}$ parameter, but we expect our method will generalize to multiple scalar fields and to moments of arbitrarily high order. We present our expressions in a field-space covariant form which we postulate to be valid for any number of fields.
\end{abstract}

Keywords: Inflation, Cosmological perturbation theory, Physics of the early universe, Quantum field theory in curved spacetime. 


\section{Introduction}

Inflation generically predicts a primordial spectrum of density perturbations which is almost precisely Gaussian [1, 2, 3, 4, 5]. In recent years the small non-Gaussian component [6, 7, 8, 9, 10, 11, 12] has emerged as an important observable [13], and will be measured with good precision by the Planck Surveyor satellite [14]. In the near future, as observational data become more plentiful, it will be important to understand the non-Gaussian signal expected in a wide variety of models, and to anticipate what conclusions can be drawn about early-universe physics from a prospective detection of primordial non-Gaussianity.

In this paper, we present a novel method for calculating the primordial nonGaussianity produced by super-horizon evolution in two-field models of inflation. Our method is based on the real-space distribution of inflationary field values on a flat hypersurface, which can be thought of as a probability density function whose evolution is determined by a form of the collisionless Boltzmann equation. Using a cumulant representation [15, 16, 17, 18] to expand our density function around an exact Gaussian, we derive ordinary differential equations which evolve the moments of this distribution. Further, we show how these moments are related to observable quantities, such as the dimensionless bispectrum measured by $f_{\mathrm{NL}}$ [14, 10]. We present numerical results which show that this method gives good agreement with other techniques. It is not necessary to make any assumptions about the inflationary model beyond requiring a canonical kinetic term and applying the slow-roll approximation. While there are already numerous methods for computing the super-horizon contribution to $f_{\mathrm{NL}}$, including the widely used $\delta N$ formalism, we believe the one reported here has a number of advantages.

First, this new technique is ideally suited to unraveling the various contributions to $f_{\mathrm{NL}}$. This is because we follow the moments of the inflaton distribution directly, which makes it straightforward to identify large contributions to the skewness or other moments. The evolution equation for each moment is simple and possesses clearly identifiable source terms, which are related to the properties of the inflationary flow on field space. This offers a clear separation between two key sources of primordial non-Gaussianity. One of these is the intrinsic non-linearity associated with evolution of the probability density function between successive flat hypersurfaces; the other is a gauge transformation from field fluctuations to the curvature peturbation, $\zeta$. It would be difficult or impossible to observe this split within the context of other calculational schemes, such as the conventional $\delta N$ formalism.

A second advantage of our method is connected with the computational cost of numerical implementation. Analytic formulas for $f_{\mathrm{NL}}$ are known in certain cases, mostly in the context of the $\delta N$ framework, but only for very specific choices of the potential [19, 20, 21, 22] or Hubble rate [23, 24]. These formulas become increasingly cumbersome as the number of fields increases, or if one studies higher moments [25, 26]. In the future, it seems clear that studies of complex models with many fields will increasingly rely on numerical methods. The numerical $\delta N$ framework requires the solution to a 
number of ordinary differential equations which scales exponentially with the number of fields. Since some models include hundreds of fields, this may present a significant obstacle [27]. Moreover, the $\delta N$ formalism depends crucially on a numerical integration algorithm with low noise properties, since finite differences must be extracted between perturbatively different initial conditions after $\sim 60$ e-folds of evolution. Thus, the background equations must be solved to great accuracy, since any small error has considerable scope to propagate.

In this paper we ultimately solve our equations numerically to determine the evolution of moments in specific models. Our method requires the solution to a number of differential equations which scales at most polynomially (or in certain cases perhaps even linearly) with the number of fields. It does not rely on extracting finite differences, and therefore is much less susceptible to numerical noise. These advantages may be shared with other schemes, such as the numerical method recently employed by Lehners \& Renaux-Petel [28].

A third advantage, to which we hope to return in a future publication, is that our formalism yields explicit evolution equations with source terms. From an analysis of these source terms, we hope that it will be possible to identify those physical features of specific models which lead to the production of large non-Gaussianities.

This paper is organized as follows. In §2, we show how the non-Gaussian parameter $f_{\mathrm{NL}}$ can be computed in our framework. The calculation remains in real space throughout (as opposed to Fourier space), which modifies the relationship between $f_{\mathrm{NL}}$ and the multi-point functions of the inflaton field. Our expression for $f_{\mathrm{NL}}$ shows a clean separation between different contributions to non-Gaussianity, especially between the intrinsic nonlinearity of the field evolution and the gauge transformation between comoving and flat hypersurfaces. In $§ 3$, we introduce our model for the distribution of inflaton field values, which is a "moment expansion" around a purely Gaussian distribution. We derive the equations which govern the evolution of the moments of this distribution in the one- and two-field cases. In \$4, we present a comparison of our new technique and those already in the literature. We compute $f_{\mathrm{NL}}$ numerically in several two-field models, and find excellent agreement between techniques. We conclude in $\$ 5$.

Throughout this paper, we use units in which $c=\hbar=1$, and the reduced Planck mass $M_{\mathrm{P}}^{-2} \equiv 8 \pi G$ is set to unity.

\section{Frameworks for computing $f_{\mathrm{NL}}$}

In this section, we introduce our new method for computing the non-Gaussianity parameter $f_{\mathrm{NL}}$. This method requires three main ingredients: a formula for the curvature perturbation, $\zeta$, in terms of the field values on a spatially flat hypersurface; expressions for the derivatives of the number of e-foldings, $N$, as a function of field values at horizon exit; and a prescription for evolving the field distribution from horizon exit to the time when we require the statistical properties of $\zeta$. The first two ingredients are given in 
Eqs. (9) $-(10)$ and (27)-(29), found at the end of $\$ 2.2$ and $\$ 2.3$ respectively. The final ingredient is discussed in $\$ 3$.

\subsection{Calculations beyond linear order}

Once it became clear that non-linearities of the microwave background anisotropies could be detected by the WMAP and Planck survey satellites [14, many authors studied higher-order correlations of the curvature perturbation. In early work, direct calculations of a correlation function were matched to the known limit of local nongaussianity [9, 10, 29, 30, 31, 32]. This method works well if isocurvature modes are absent, so that the curvature perturbation is constant after horizon exit. In the more realistic situation that isocurvature modes cause evolution on superhorizon scales, all correlation functions become time dependent. Various formalisms have been employed to describe this evolution. Lyth \& Rodríguez [11] extended the $\delta N$ method [33, 34] beyond linear order. This method is simple and well-suited to analytical calculation. Rigopoulos, Shellard and van Tent [35, 36] worked with a gradient expansion, rewriting the field equations in Langevin form. The noise term was used as a proxy for setting initial conditions at horizon crossing. A similar 'exact' gradient formalism was written down by Langlois \& Vernizzi [37, 38, 39. In its perturbative form, this approach has been used by Lehners \& Renaux-Petel to obtain numerical results [28]. Another numerical scheme has been introduced by Huston \& Malik [40].

What properties do we require of a successful prediction? Consider a typical observer, drawn at random from an ensemble of realizations of inflation. In any of the formalisms discussed above, we aim to estimate the statistical properties of the curvature perturbation which would be measured by such an observer. Some realizations may yield statistical properties which are quite different from the ensemble average, but these large excursions are uninteresting unless anthropic arguments are in play.

Next we introduce a collection of comparably-sized spacetime volumes whose mutual scatter is destined to dominate the microwave background anisotropy on a given scale. Neglecting spatial gradients, each spacetime volume will follow a trajectory in field space which is slightly displaced from its neighbors. The scatter between trajectories is determined by initial conditions set at horizon exit, which are determined by promoting the vacuum fluctuation to a classical perturbation $\dagger \mathrm{A}$ correct prediction is a function of the trajectories followed by every volume in the collection, taken as a whole. One never makes a prediction for a single trajectory.

Each spacetime volume follows a trajectory, which we label with its position $\varphi^{\star}$

$\dagger$ The scatter among trajectories may grow with the overall volume, owing to back-reaction effects in de Sitter space 41, 42, 43, 44. If inflation can end in more than one vacuum, leading to different predictions for observable quantities, it may be helpful to evaluate this enhanced dispersion for the purpose of deciding into which vacuum the field will fall. Once this minimum has been decided, however, large-volume effects needlessly complicate the calculation. In this paper, we will always assume that predictions are being made by following a sufficient number of trajectories to determine the statistical properties with reasonable precision, but no more. 
at some fixed time, to be made precise below. Throughout this paper, superscript ' $\star$ ' denotes evaluation on a spatially flat hypersurface. Consider the evolution of some quantity of interest, $F$, which is a function of trajectory. If we know the distribution $P\left(\varphi^{\star}\right)$ we can study statistical properties of $F$ such as the $m^{\text {th }}$ moment $\kappa_{m}$,

$$
\kappa_{m} \equiv \int \mathrm{d} \varphi^{\star} P\left(\varphi^{\star}\right)\left[F\left(\varphi^{\star}\right)-\langle F\rangle\right]^{m}
$$

where we have introduced the ensemble average of $F$,

$$
\langle F\rangle \equiv \int \mathrm{d} \varphi^{\star} P\left(\varphi^{\star}\right) F\left(\varphi^{\star}\right)
$$

In Eqs. (11)-(2), $\varphi^{\star}$ stands for a collection of any number of fields. It is the $\kappa_{m}$ which are observable quantities.

Eq. (11) defines what we will call the exact separate universe picture. It is often convenient to expand $F\left(\varphi^{\star}\right)$ as a power series in the field values centered on a fiducial trajectory, labelled 'fid,'

$$
F\left(\varphi^{\star}\right)-F\left(\varphi_{\text {fid }}^{\star}\right)=\left.\sum_{n=1}^{\infty} \frac{1}{n !}\left(\varphi^{\star}-\varphi_{\text {fid }}^{\star}\right)^{n} \frac{\partial^{n} F}{\partial\left(\varphi^{\star}\right)^{n}}\right|_{\varphi^{\star}=\varphi_{\text {fid }}^{\star}} .
$$

When Eq. (3) is used to evaluate the $\kappa_{m}$, we refer to the 'perturbative' separate universe picture. If all terms in the power series are retained, these two versions of the calculation are formally equivalent. In unfavorable cases, however, convergence may occur slowly or not at all. This possibility was discussed in Refs. [45, 46]. Although our calculation is formally perturbative, it is not directly equivalent to Eq. (3). We briefly discuss the relation of our calculation to conventional perturbation theory in $\$ 5$.

\subsection{Calculating $f_{\mathrm{NL}}$ in the separate universe picture}

By definition, the curvature perturbation $\zeta$ measures local fluctuations in expansion history (expressed in e-folds $N$ ), calculated on a comoving hypersurface. In many models, the curvature perturbation is synthesized by superhorizon physics, which reprocesses a set of Gaussian fluctuations generated at horizon exit. In a single-field

model, only one Gaussian fluctuation can be present, which we label $\zeta_{g}$. Neglecting spatial gradients, the total curvature perturbation must then be a function of $\zeta_{g}$ alone. For $\left|\zeta_{g}\right| \ll 1$, this can be well-approximated by

$$
\zeta \simeq \zeta_{g}+\frac{3}{5} f_{\mathrm{NL}}\left(\zeta_{g}^{2}-\left\langle\zeta_{g}^{2}\right\rangle\right)
$$

where $f_{\mathrm{NL}}$ is independent of spatial position. Eq. (4) defines the so-called "local" form of non-gaussianity. It applies only when quantum interference effects can be neglected, making $\zeta$ a well-defined object rather than a superposition of operators [47]. If this condition is satisfied, spatial correlations of $\zeta$ may be extracted and it follows that $f_{\mathrm{NL}}$ can be estimated using the rule

$$
f_{\mathrm{NL}} \simeq \frac{5}{18} \frac{\langle\zeta \zeta \zeta\rangle}{\langle\zeta \zeta\rangle^{2}}
$$


where we have recalled that $\zeta$ is nearly Gaussian, or equivalently that $\left|f_{\mathrm{NL}}\right| \ll\left|\zeta_{g}\right|^{-1}$.

With $f_{\mathrm{NL}}$ spatially independent, Eq. (44) strictly applies only in single-field inflation. In this case one can accurately determine $f_{\mathrm{NL}}$ by applying Eq. (4) to a single trajectory with fixed initial conditions, as in the method of Lehners \& Renaux-Petel [28]. Where more than one field is present, $f_{\mathrm{NL}}$ may vary in space because it depends on the isocurvature modes. In this case one must determine $f_{\mathrm{NL}}$ statistically on a bundle of adjacent trajectories which sample the local distribution of isocurvature modes. Eq. (5) is then indispensible. Following Maldacena [10], and later Lyth \& Rodríguez [11], we adopt Eq. (5) as our definition of $f_{\mathrm{NL}}$, whatever its origin. In real space, the coefficient $5 / 18$ in Eq. (5) depends on the convention $\langle\zeta\rangle=0$. More generally, this follows from the definition of $\kappa_{m}$, Eq. (11). In Fourier space, either prescription is automatically enforced after dropping disconnected contributions, again leading to Eq. (5).

To proceed, we require estimates of the correlation functions $\langle\zeta \zeta\rangle$ and $\langle\zeta \zeta \zeta\rangle$. We first describe the conventional approach, in which ' $\star$ ' denotes a flat hypersurface at a fixed initial time. The quantity $N\left(\varphi_{i}^{\star}\right)$ denotes the number of e-foldings between this initial slice and a final comoving hypersurface, where $i$ indexes the species of light scalar fields. The local variation in expansion can be written in the fiducial picture as

$$
\zeta(\mathbf{x}) \equiv \delta N(\mathbf{x})=\left.\sum_{n=1}^{\infty} \frac{1}{n !} \frac{\partial^{n} N\left(\varphi_{i}^{\star}\right)}{\partial \varphi_{j_{1}}^{\star} \cdots \partial \varphi_{j_{n}}^{\star}}\right|_{\varphi_{i}^{\star}=\varphi_{i, \mathrm{fid}}^{\star}} \delta \varphi_{j_{1}}^{\star}(\mathbf{x}) \cdots \delta \varphi_{j_{n}}^{\star}(\mathbf{x}),
$$

where $\delta \varphi_{j}^{\star} \equiv \varphi_{j}^{\star}-\varphi_{j, \text { fid }}^{\star}$.

Subject to the condition that the relevant scales are all outside the horizon, we are free to choose the initial time - set by the hypersurface ' $\star$ ' - at our convenience. In the conventional approach, ' $\star$ ' is taken to lie a few e-folds after our collection of spacetime volumes passes outside the causal horizon [11, 12]. This choice has many virtues. First, we need to know statistical properties of the field fluctuations $\delta \varphi_{i}^{\star}$ only around the time of horizon crossing, where they can be computed without the appearance of large logarithms [44, 48]. Second, as a consequence of the slow-roll approximation, the $\delta \varphi_{i}^{\star}$ are uncorrelated at this time, leading to algebraic simplifications. Finally, the $\delta N$ formula subsumes a gauge transformation from the field variables $\delta \varphi_{i}^{\star}$ to the observational variable $\zeta$. Using Eqs. (1)-(2), (5) and (6) , one finds that $f_{\mathrm{NL}}$ can be written to a good approximation [11]

$$
f_{\mathrm{NL}} \approx \frac{5}{6} \frac{N_{, i} N_{, j} N_{, i j}}{\left(N_{, k} N_{, k}\right)^{2}}
$$

‘ $\star$ ' at horizon crossing

where $N_{, i} \equiv \partial N / \partial \varphi_{i}^{\star}$ and for simplicity we have dropped the ' $\star$ ' which indicates time of evaluation. A similar definition applies for $N_{, i j}$.

Eq. (7) is accurate up to small intrinsic non-Gaussianities present in the field fluctuations at horizon exit. As a means of predicting $f_{\mathrm{NL}}$ it is pleasingly compact, and straightforward to evaluate in many models. Unfortunately, it also obscures the physics which determines $\left|f_{\mathrm{NL}}\right|$. For this reason it is hard to infer, from Eq. (77) alone, 
those classes of models in which $\left|f_{\mathrm{NL}}\right|$ is always large or small $\ddagger$

Our strategy is quite different. We choose ' $\star$ ' to lie around the time when we require the statistical properties of $\zeta$. The role of the $\delta N$ formula, Eq. ([6), is then to encode only the gauge transformation between the $\delta \varphi_{i}^{\star}$ and $\zeta$. In $\$ 2.3$ below, we show how the appropriate gauge transformation is computed using the $\delta N$ formula. In the present section we restrict our attention to determining a formula for $f_{\mathrm{NL}}$ under the assumption that the distribution of field values on ' $\star$ ' is known. In \$3, we will supply the required prescription to evolve the distribution of field values between horizon exit and ' $\star$ '.

Although the $\delta \varphi_{i}^{\star}$ are independent random variables at horizon exit, correlations can be induced by subsequent evolution. One must therefore allow for off-diagonal terms in the two-point function. Remembering that we are working with a collection of spacetime volumes in real space, smoothed on some characteristic scale, we write

$$
\left\langle\delta \varphi_{i}^{\star} \delta \varphi_{j}^{\star}\right\rangle \equiv \Sigma_{i j}
$$

$\Sigma_{i j}$ does not vary in space, but it may be a function of the scale which characterizes our ensemble of spacetime volumes. In all but the simplest models it will vary in time. It is also necessary to account for intrinsic non-linearities among the $\delta \varphi_{i}^{\star}$, which are small at horizon crossing but may grow. We define

$$
\left\langle\delta \varphi_{i}^{\star} \delta \varphi_{j}^{\star} \delta \varphi_{k}^{\star}\right\rangle \equiv \alpha_{i j k} .
$$

Likewise, $\alpha_{i j k}$ should be regarded as a function of time and scale. The permutation symmetries of an expectation value such as (9) guarantee that, for example, $\alpha_{122}=$ $\alpha_{212}=\alpha_{221} \oint$ When written explicitly, we place the indices of symbols such as $\alpha$ in numerical order. Neglecting a small $\left(\lesssim \mathrm{O}\left(\Sigma^{3}\right)\right)$ intrinsic four-point correlation, it follows that

$$
\left\langle\delta \varphi_{i}^{\star} \delta \varphi_{j}^{\star} \delta \varphi_{k}^{\star} \delta \varphi_{m}^{\star}\right\rangle=\Sigma_{i j} \Sigma_{k m}+\Sigma_{i k} \Sigma_{j m}+\Sigma_{i m} \Sigma_{j k} .
$$

Now we specialize to a two-field model, parametrized by fields $\varphi_{1}$ and $\varphi_{2}$. Using Eqs. (11)-(2), (6) and (8)), it follows that the two-point function of $\zeta$ satisfies

$$
\langle\zeta \zeta\rangle=N_{, 1}^{2} \Sigma_{11}+N_{, 2}^{2} \Sigma_{22}+2 N_{, 1} N_{, 2} \Sigma_{12} \quad \text { ‘' arbitrary }
$$

The three-point function can be written

$$
\langle\zeta \zeta \zeta\rangle=\langle\zeta \zeta \zeta\rangle_{1}+\langle\zeta \zeta \zeta\rangle_{2}
$$

where we have identified two separate contributions, labelled ' 1 ' and ' 2 '. The ' 1 ' term includes all contributions involving intrinsic non-linearities, those which arise from nonGaussian correlations among the field fluctuations,

$\langle\zeta \zeta \zeta\rangle_{1}=N_{, 1}^{3} \alpha_{111}+N_{, 2}^{3} \alpha_{222}+3 N_{, 1}^{2} N_{, 2} \alpha_{112}+3 N_{, 1} N_{, 2}^{2} \alpha_{122} . \quad$ ‘' arbitrary

$\ddagger$ Even in simple models it can be quite subtle to determine what range of $\left|f_{\mathrm{NL}}\right|$ is dynamically allowed. See, for example, Refs. 21, 22].

$\S$ We are assuming that these expectation values are ensemble averages over classical stochastic fields, and are therefore invariant under reordering of the fields. 
The ' 2 ' term encodes non-linearities arising directly from the gauge transformation to $\zeta$

$$
\begin{aligned}
\langle\zeta \zeta \zeta\rangle_{2}=\frac{9}{2} N_{, 1}^{2} & N_{, 11} \Sigma_{11}^{2}+\frac{9}{2} N_{, 2}^{2} N_{, 22} \Sigma_{22}^{2} \\
& +9\left(N_{, 1} N_{, 2} N_{, 11}+N_{, 1}^{2} N_{, 12}\right) \Sigma_{11} \Sigma_{12}+9\left(N_{, 1} N_{, 2} N_{, 22}+N_{, 2}^{2} N_{, 12}\right) \Sigma_{12} \Sigma_{22} \\
& +\frac{3}{2}\left(N_{, 2}^{2} N_{, 11}+N_{, 1}^{2} N_{, 22}+4 N_{, 1} N_{, 2} N_{, 12}\right)\left(\Sigma_{11} \Sigma_{22}+2 \Sigma_{12}^{2}\right) \\
& -\frac{3}{2}\left(N_{, 11} \Sigma_{11}+2 N_{, 12} \Sigma_{12}+N_{, 22} \Sigma_{22}\right)\langle\zeta \zeta\rangle,
\end{aligned}
$$

After use of Eq. (5) , Eqs. (12)-(14) can be used to extract the non-linearity parameter $f_{\mathrm{NL}}$. This decomposes likewise into two contributions $f_{\mathrm{NL}}=f_{\mathrm{NL} 1}+f_{\mathrm{NL} 2}$, which we shall discuss in more detail in $\$ 4$.

\subsection{The derivatives of $N$}

To compute $f_{\mathrm{NL}}$ in concrete models, we require expressions for the derivatives $N_{, i}$ and $N_{, i j}$. For generic initial and final times, these are difficult to obtain. Lyth \& Rodríguez [11] used direct integration, which is effective for quadratic potentials and constant slowroll parameters. Vernizzi \& Wands [19] obtained expressions in a two-field model with an arbitrary sum-separable potential by introducing Gaussian normal coordinates on the space of trajectories. Their approach was generalized to many fields by Battefeld \& Easther [20]. Product-separable potentials can be accommodated using the same technique [49]. An alternative technique has been proposed by Yokoyama et al. [50].

A considerable simplification occurs in the present case, because we only require the derivative evaluated between flat and comoving hypersurfaces which coincide in the unperturbed universe. For any species $i$, and to leading order in the slow-roll

approximation, the number of e-folds $N$ between the flat hypersurface ' $\star$ ' and a comoving hypersurface ' $c$ ' satisfies

$$
N \equiv-\int_{\varphi_{i}^{\star}}^{\varphi_{i}^{c}} \frac{V}{V_{, i}} \mathrm{~d} \varphi_{i} \quad \text { no sum on } i
$$

where $V_{, i} \equiv \partial V / \partial \varphi_{i}$ and $\left\{\varphi_{i}^{\star}, \varphi_{i}^{c}\right\}$ are the field values evaluated on ' $\star$ ' and ' $c$,' respectively. Under an infinitesimal shift of $\varphi_{i}^{\star}$, we deduce that $N_{, i}$ obeys

$$
N_{, i}=\left(\frac{V}{V_{, i}}\right)^{\star}-\left(\frac{V}{V_{, i}}\right)^{c} \frac{\partial \varphi_{i}^{c}}{\partial \varphi_{i}^{\star}} \quad \text { no sum on } i .
$$

Note that this applies for an arbitrary $V$, which need not factorize into a sum or product of potentials for the individual species $i$. In principle a contribution from variation of the integrand is present, which spoils a naïve attempt to generalize the method of Refs. [19, 20, 49] to an arbitrary potential. This contribution vanishes in virtue of our supposition that ' $\star$ ' and ' $c$ ' are infinitesimally separated.

To compute $\partial \varphi_{i}^{c} / \partial \varphi_{j}^{\star}$ it is helpful to introduce a quantity $C$, which in the sumseparable case coincides with the conserved quantity of Vernizzi \& Wands [19, 51]. For our specific choice of a two-field model, this takes the form

$$
C\left(\varphi_{1}, \varphi_{2}\right) \equiv \int^{\varphi_{1}} \frac{H^{2}}{V_{, 1}} \mathrm{~d} \varphi_{1}^{\prime}-\int^{\varphi_{2}} \frac{H^{2}}{V_{, 2}} \mathrm{~d} \varphi_{2}^{\prime}
$$


where the integrals are evaluated on a single spatial hypersurface. In an $M$-field model, one would obtain $M-1$ conserved quantities which label the isocurvature fields. The construction of these quantities is discussed in Refs. [20, 25]. For sum-separable potentials one can show using the equations of motion that $C$ is conserved under time evolution to leading order in slow-roll. It is not conserved for general potentials, but the variation can be neglected for infinitesimally separated hypersurfaces.

Under a change of trajectory, $C$ varies according to the rules

$$
\frac{\partial C}{\partial \varphi_{1}^{\star}}=\frac{H^{2}}{V_{, 1}}
$$

and

$$
\frac{\partial C}{\partial \varphi_{2}^{\star}}=-\frac{H^{2}}{V_{, 2}} .
$$

The comoving hypersurface ' $c$ ' is defined by

$$
\frac{1}{2}\left(\dot{\varphi}_{1}^{2}+\dot{\varphi}_{2}^{2}\right)+V=\text { constant }
$$

We are assuming that the slow-roll approximation applies, so that the kinetic energy may be neglected in comparison with the potential $V$. Therefore on ' $c$ ' we have

$$
\frac{\partial V}{\partial \varphi_{1}^{c}} \frac{\partial \varphi_{1}^{c}}{\partial C}+\frac{\partial V}{\partial \varphi_{2}^{c}} \frac{\partial \varphi_{2}^{c}}{\partial C}=0
$$

Combining Eqs. (18), (19) and (21) we obtain expressions for $\partial \varphi_{i}^{c} / \partial \varphi_{j}^{\star}$, namely

$$
\begin{aligned}
& \frac{\partial \varphi_{1}^{c}}{\partial \varphi_{1}^{\star}}=\left(\frac{V_{, 1}}{V}\right)^{c}\left(\frac{V}{V_{, 1}}\right)^{\star} \sin ^{2} \theta, \\
& \frac{\partial \varphi_{1}^{c}}{\partial \varphi_{2}^{\star}}=-\left(\frac{V_{, 1}}{V}\right)^{c}\left(\frac{V}{V_{, 2}}\right)^{\star} \sin ^{2} \theta, \\
& \frac{\partial \varphi_{2}^{c}}{\partial \varphi_{1}^{\star}}=-\left(\frac{V_{, 2}}{V}\right)^{c}\left(\frac{V}{V_{, 1}}\right)^{\star} \cos ^{2} \theta, \\
& \frac{\partial \varphi_{2}^{c}}{\partial \varphi_{2}^{\star}}=\left(\frac{V_{, 2}}{V}\right)^{c}\left(\frac{V}{V_{, 2}}\right)^{\star} \cos ^{2} \theta,
\end{aligned}
$$

where we have defined

$$
\tan ^{2} \theta \equiv \frac{\left(V_{, 2}\right)^{2}}{\left(V_{, 1}\right)^{2}}
$$

Eqs. (22) -(25) can alternatively be derived without use of $C$ by comparing Eq. (16) with the formulas of Ref. [52], which were derived using conventional perturbation theory. Applying (16), we obtain

$$
N_{, 1}=\left(\frac{V}{V_{, 1}}\right)^{\star} \cos ^{2} \theta ; \quad N_{, 2}=\left(\frac{V}{V_{, 2}}\right)^{\star} \sin ^{2} \theta .
$$

To proceed, we require the second derivatives of $N$. These can be obtained directly from (27), after use of Eqs. (22)-(25). We find

$$
\begin{aligned}
N_{, 11}=[1- & \left.\frac{V V_{, 11}}{\left(V_{, 1}\right)^{2}}\right]^{\star} \cos ^{2} \theta+2\left(\frac{V}{V_{, 1}}\right)^{\star 2} \cos ^{2} \theta \\
& \times\left[\frac{V_{, 11}}{V} \sin ^{2} \theta-\frac{V_{, 1} V_{, 12}}{V V_{, 2}} \sin ^{4} \theta-\left(\frac{V_{, 11}}{V}-\frac{V_{, 22}}{V}+\frac{V_{, 2} V_{, 12}}{V V_{, 1}}\right) \cos ^{2} \theta \sin ^{2} \theta\right]^{c} .
\end{aligned}
$$


An analogous expression for $N_{, 22}$ can be obtained after the simultaneous exchange $\{1 \leftrightarrow 2, \sin \leftrightarrow \cos \}$. The mixed derivative satisfies

$$
\begin{aligned}
N_{, 12}=2( & \left.\frac{V}{V_{, 1}}\right)^{\star}\left(\frac{V}{V_{, 2}}\right)^{\star} \cos ^{2} \theta \\
\times & {\left[-\frac{V_{, 11}}{V} \sin ^{2} \theta+\frac{V_{, 1} V_{, 12}}{V V_{, 2}} \sin ^{4} \theta+\left(\frac{V_{, 11}}{V}-\frac{V_{, 22}}{V}+\frac{V_{, 2} V_{, 12}}{V V_{, 1}}\right) \cos ^{2} \theta \sin ^{2} \theta\right]^{c} } \\
& +\cos ^{2} \theta\left(\frac{V_{, 2}}{V_{, 1}}-\frac{V V_{, 12}}{V_{, 1}^{2}}\right)^{c} .
\end{aligned}
$$

Now that the calculation is complete, we can drop the superscripts ' $\star$ ' and ' $c$,' since any background quantity is the same on either hypersurface. Once this is done it can be verified that (despite appearances) Eq. (29) is invariant under the exchange $1 \leftrightarrow 2$.

\section{Transport equations}

In this section we return to the problem of evolution between horizon exit and the time of observation, and supply the prescription which connects the distribution of field values at these two times.

\subsection{Non-gaussian distribution in the single-field case}

We begin by discussing the single-field system, which lacks the technical complexity of the two-field case, yet still exhibits certain interesting features which recur there. Among these features are the subtle difference between motion of the statistical mean and the background field value, and the hierarchy of moment evolution equations. Moreover, the structure of the moment mixing equations is similar to that which obtains in the two-field case. For this reason, the one-field scenario provides an instructive example of the techniques we wish to employ.

Recall that we work in real space with a collection of comparably sized spacetime volumes, each with a slightly different expansion history, and the scatter in these histories determines the microwave background anisotropy on a given angular scale. Within each volume the smoothed background field $\varphi$ takes a uniform value described by a density function $P(\varphi)$, where in this section we are dropping the superscript ' $\star$ ' denoting evaluation of spatially flat hypersurfaces. Our ultimate goal is to calculate the reduced bispectrum, $f_{\mathrm{NL}}$, which describes the third moment of $P(\varphi)$. In the language

of probability this is the skewness, which we denote $\alpha$. A Gaussian distribution has skewness zero, and inflation usually predicts that the skew is small. For this reason, rather than seek a distribution with non-zero third moment, as proposed in Ref. [18], we will introduce higher moments as perturbative corrections to the Gaussian. Such a procedure is known as a cumulant expansion.

The construction of cumulant expansions is a classical problem in probability theory. We seek a distribution with centroid $\varphi_{0}$, variance $\sigma^{2}$, and skew $\alpha$, with all higher 
moments determined by $\sigma$ and $\alpha$ alone. A distribution with suitable properties is

$$
P(\varphi)=P_{g}(\varphi)\left[1+\frac{\alpha}{6 \sigma^{3}} H_{3}\left(\frac{\varphi-\varphi_{0}}{\sigma}\right)\right]
$$

where

$$
P_{g}(\varphi) \equiv \frac{1}{\sqrt{2 \pi} \sigma} \exp \left[-\frac{\left(\varphi-\varphi_{0}\right)^{2}}{2 \sigma^{2}}\right]
$$

is a pure Gaussian and $H_{n}$ denotes the $n^{\text {th }}$ Hermite polynomial, for which there are multiple normalization conventions. We choose to normalize so that

$$
\int_{-\infty}^{\infty} \frac{1}{\sqrt{2 \pi}} e^{-x^{2} / 2} H_{n}(x) H_{m}(x) \mathrm{d} x=n ! \delta_{m n}
$$

which implies that the leading term of $H_{n}(x)$ is $x^{n}$. This is sometimes called the "Probabilist's convention." We define expectation values $\langle\cdots\rangle$ by the usual rule,

$$
\langle F\rangle \equiv \int_{-\infty}^{\infty} P(\varphi) F \mathrm{~d} x .
$$

The probability density function in Eq. (30) has the propertiest

$$
\langle 1\rangle=1, \quad\langle\varphi\rangle=\varphi_{0}, \quad\left\langle\left(\varphi-\varphi_{0}\right)^{2}\right\rangle=\sigma^{2}, \quad \text { and } \quad\left\langle\left(\varphi-\varphi_{0}\right)^{3}\right\rangle=\alpha .
$$

The moments $\varphi_{0}, \sigma$, and $\alpha$ may be time-dependent, so evolution of the probability density in time can be accommodated by finding evolution equations for these quantities.

The density function given in Eq. (30) is well-known and has been applied in many situations. It is a solution to the problem of approximating a nearly-Gaussian distribution whose moments are known. Eq. (30) is in fact the first two terms of the Gram-Charlier 'A' series, also sometimes called the Gauss-Hermite series $₫$ In recent years it has found multiple applications to cosmology, of which our method is closest to that of Taylor \& Watts [53]. Other applications are discussed in Refs. [16, 17, 15, 54, 18, 53, 55, 56, 57, 58, 59, 60]. For a review of the 'A' series and related nearly-Gaussian probability distributions from an astrophysical perspective, see [61]. In this paper, we will refer to Eq. (30) and its natural generalization to higher moments as the "moment expansion."

In the slow-roll approximation, the field in each spacetime volume obeys a simple equation of motion

$$
\frac{\mathrm{d} \varphi}{\mathrm{d} N}=-\frac{\partial \ln V(\varphi)}{\partial \varphi} \equiv u(\varphi)
$$

$\dagger$ These formulas apply for arbitrary values of $\alpha$, and do not depend on the approximation that $\alpha$ is small. However, for large $\alpha$ the density function (30) may become negative for some values of $\varphi$. It then ceases to be a probability density in the strict sense. This does not present a problem in practice, since we are interested in distributions which are approximately Gaussian, and for which $\alpha$ will typically be small. Moreover, our principal use of Eq. (30) is as a formal tool to extract evolution equations for each moment. For this reason we will not worry whether $P(\varphi)$ defines an honest probability density function in the strict mathematical sense.

$\ddagger$ In the physics literature, this series has sometimes erroneously been called the Edgeworth expansion. 
where $N$ records the number of e-foldings of expansion. We refer to $u(\varphi)$ as the velocity field. Expanding $u$ about the instantaneous centroid $\varphi_{0}$ gives

$$
u(\varphi)=u_{0}+u_{\varphi}\left(\varphi-\varphi_{0}\right)+\frac{1}{2} u_{\varphi \varphi}\left(\varphi-\varphi_{0}\right)^{2}+\cdots,
$$

where

$$
\left.u_{0} \equiv u\right|_{\varphi_{0}},\left.\quad u_{\varphi} \equiv \frac{\mathrm{d} u}{\mathrm{~d} \varphi}\right|_{\varphi_{0}},\left.\quad u_{\varphi \varphi} \equiv \frac{\mathrm{d}^{2} u}{\mathrm{~d} \varphi^{2}}\right|_{\varphi_{0}} .
$$

The value of $\varphi_{0}$ evolves with time, so each expansion coefficient is time-dependent. Hence, we do not assume that the velocity field is globally well-described by a quadratic Taylor expansion, but merely that it is well-described as such in the neighborhood of the instantaneous centroid. We expand the velocity field to second order, although in principle this expansion could be carried to arbitrary order.

It remains to specify how the probability density evolves in time. Conservation of probability leads to the transport equation

$$
\frac{\partial P}{\partial N}+\frac{\partial}{\partial \varphi}(u P)=0
$$

Eq. (38) can also be understood as the limit of a Chapman-Kolmogorov process as the size of each hop goes to zero. It is well known-for example, from the study of Starobinsky's diffusion equation which forms the basis of the stochastic approach to inflation [62] - that the choice of time variable in this equation is significant, with different choices corresponding to the selection of a temporal gauge. We have chosen to use the e-folding time, $N$, which means that we are evolving the distribution on hypersurfaces of uniform expansion. These are the spatially flat hypersurfaces whose field perturbations enter the $\delta N$ formulas described in $\$ 2$.

In principle, Eq. (38) can be solved directly. In practice it is simpler to extract equations for the moments of $P$, giving evolution equations for $\varphi_{0}, \sigma$ and $\alpha$. To achieve this, one need only resolve Eq. (38) into a Hermite series of the form

$$
P_{g} \sum_{n \geqslant 0} c_{n} H_{n}\left(\varphi-\varphi_{0}\right)=0
$$

The Hermite polynomials are linearly independent, and application of the orthogonality condition (32) shows that the $c_{n}$ must all vanish. This leads to a hierarchy of equations $c_{n}=0$, which we refer to as the moment hierarchy. At the top of the hierarchy, the equation $c_{0}=0$ is empty and expresses conservation of probability.

The first non-trivial equation requires $c_{1}=0$ and yields an evolution equation for the centroid $\varphi_{0}$,

$$
\frac{\mathrm{d} \varphi_{0}}{\mathrm{~d} N}=u_{0}+\frac{1}{2} u_{\varphi \varphi} \sigma^{2}
$$

The first term on the right-hand side drives the centroid along the velocity field, as one would anticipate based on the background equation of motion, Eq. (35). However, the second term shows that the centroid is also influenced as the wings of the probability distribution probe the nearby velocity field. This influence is not captured by the 
background equation of motion. If we are in a situation with $u_{\varphi \varphi}>0$, then the wings of the density function will be moving faster than the center. Hence, the velocity of the centroid will be larger than one might expect by restricting attention to $\varphi_{0}$. Accordingly, the mean fluctuation value is not following a solution to the background equations of motion.

Evolution equations for the variance $\sigma^{2}$ and skew $\alpha$ are obtained after enforcing $c_{2}=c_{3}=0$, yielding

$$
\begin{aligned}
& \frac{\mathrm{d} \sigma^{2}}{\mathrm{~d} N}=2 u_{\varphi} \sigma^{2}+u_{\varphi \varphi} \alpha \\
& \frac{\mathrm{d} \alpha}{\mathrm{d} N}=3 u_{\varphi} \alpha+3 u_{\varphi \varphi} \sigma^{4}
\end{aligned}
$$

In both equations, the first term on the right-hand sides describes how $\sigma$ and $\alpha$ scale as the density function expands or contracts in response to the velocity field. These terms force $\sigma^{2}$ and $\alpha$ to scale in proportion to the velocity field. Specifically, if we temporarily drop the second terms in each equation above, one finds that $\sigma^{2} \sim u^{2}$ and $\alpha \sim u^{3}$. This precisely matches our expectation for the scaling of these quantities. Hence, these terms account for the Jacobians associated with infinitesimal transformations induced by the flow $u(\varphi)$.

For applications to inflationary non-Gaussianity, the second terms in (41) and (42) are more relevant. These terms describe how each moment is sourced by higher moments and the interaction of the density function with the velocity field. In the example above, if we are in a situation where $u_{\varphi \varphi}>0$, the tails of the density function are moving faster than the core. This means that one tail is shrinking and the other is extending, skewing the probability density. The opposite occurs when $u_{\varphi \varphi}<0$. These effects are measured by the second term in (42). Hence, by expanding our PDF to the third moment, and our velocity field to quadratic order, we are able to construct a set of evolution equations which include the leading-order source terms for each moment.

\subsection{The two-field case}

There is little conceptually new as we move from one field to two. The new features are mostly technical in nature. Our primary challenge is a generalization of the moment expansion to two fields, allowing for the possibility of correlation between the fields. With this done, we can write down evolution equations whose structure is very similar to those found in the single-field case.

The two-field system is described by a two-dimensional velocity field $u_{i}$, defined by

$$
u_{i}=\frac{\mathrm{d} \varphi_{i}}{\mathrm{~d} N},
$$

where again we are using the number of e-folds $N$ as the time variable. The index $i$ takes values in $\{1,2\}$. While we think it is likely that our equations generalize to any number of fields, we have only explicitly constructed them for a two-field system. As will become clear below, certain steps in this construction apply only for two fields, and hence we make no claims at present concerning examples with three or more fields. 
The two-dimensional transport equation is

$$
\frac{\partial P\left(\varphi_{i}, N\right)}{\partial N}+\frac{\partial}{\partial \varphi_{j}}\left[u_{j} P\left(\varphi_{i}, N\right)\right]=0 .
$$

Here and in the following we have returned to our convention that repeated species indices are summed. As in the single-field case, we construct a probability distribution which is nearly Gaussian, but has a small non-zero skewness. That gives

$$
P\left(\varphi_{i}, N\right) \equiv P_{g}\left(\varphi_{i}, N\right) P_{n g}\left(\varphi_{i}, N\right)
$$

where $P_{g}$ is a pure Gaussian distribution, defined by

$$
P_{g}\left(\varphi_{i}, N\right)=\frac{1}{2 \pi \sqrt{\operatorname{det} \Sigma}} \exp \left[-\frac{1}{2}\left(\varphi_{i}-\Phi_{i}\right)\left(\Sigma^{-1}\right)_{i j}\left(\varphi_{j}-\Phi_{j}\right)\right] .
$$

In this equation, $\Phi_{i}$ defines the center of the distribution and $\Sigma$ describes the covariance between the fields. We adopt a conventional parametrization in terms of variances $\sigma_{i}^{2}$ and a correlation coefficient $\rho$,

$$
\Sigma \equiv\left(\begin{array}{cc}
\sigma_{1}^{2} & \rho \sigma_{1} \sigma_{2} \\
\rho \sigma_{1} \sigma_{2} & \sigma_{2}^{2}
\end{array}\right) .
$$

The matrix $\sigma$ defines two-point correlations of the fields,

$$
\left\langle\left(\varphi_{i}-\Phi_{i}\right)\left(\varphi_{j}-\Phi_{j}\right)\right\rangle=\Sigma_{i j} .
$$

All skewnesses are encoded in $P_{n g}$. Before defining this explicitly, it is helpful to pause and notice a complication inherent in Eqs. (46)-(47) which was not present in the single-field case. To extract a hierarchy of moment evolution equations from the transport equation, Eq. (38), we made the expansion given in (39) and argued that orthogonality of the Hermite polynomials implied the hierarchy $c_{n}=0$. However, Hermite polynomials of the form $H_{n}\left[\left(\varphi_{i}-\Phi_{i}\right) / \sigma\right]$ are not orthogonal under the Gaussian measure of Eq. (46). Following an expansion analogous to Eq. (39) the moment hierarchy would comprise linear combinations of the coefficients. The problem is essentially an algebraic question of Gram-Schmidt orthogonalization.

To avoid this problem it is convenient to diagonalize the covariance matrix $\Sigma$, introducing new variables $X$ and $Y$ for which Eq. (46) factorizes into the product of two measures under which the polynomials $H_{n}(X)$ and $H_{n}(Y)$ are separately orthogonal. The necessary redefinitions are

$$
X \equiv \frac{1}{\sqrt{2(1+\rho)}}\left[\left(\frac{\varphi_{1}-\Phi_{1}}{\sigma_{1}}\right)+\left(\frac{\varphi_{2}-\Phi_{2}}{\sigma_{2}}\right)\right]
$$

and

$$
Y \equiv \frac{1}{\sqrt{2(1-\rho)}}\left[\left(\frac{\varphi_{1}-\Phi_{1}}{\sigma_{1}}\right)-\left(\frac{\varphi_{2}-\Phi_{2}}{\sigma_{2}}\right)\right] .
$$

A simple expression for $P_{g}$ can be given in terms of $X$ and $Y$,

$$
P_{g}=\frac{1}{2 \pi} \exp \left(-\frac{X^{2}}{2}\right) \exp \left(-\frac{Y^{2}}{2}\right) \text {. }
$$


We now define the non-Gaussian factor, which encodes the skewnesses, to be

$$
\begin{aligned}
P_{n g} \equiv 1+\frac{\alpha_{X X X}}{6} & H_{3}(X) \\
& +\frac{\alpha_{X X Y}}{2} H_{2}(X) H_{1}(Y)+\frac{\alpha_{X Y Y}}{2} H_{1}(X) H_{2}(Y)+\frac{\alpha_{Y Y Y}}{6} H_{3}(Y) .
\end{aligned}
$$

In these variables we find $\left\langle X^{2}\right\rangle=\left\langle Y^{2}\right\rangle=1$, but $\langle X Y\rangle=0$. In addition, we have

$$
\langle X X X\rangle=\alpha_{X X X}, \quad\langle X X Y\rangle=\alpha_{X X Y}, \quad\langle X Y Y\rangle=\alpha_{X Y Y}, \quad \text { and } \quad\langle Y Y Y\rangle=\alpha_{Y Y Y} \cdot(53)
$$

In order for Eq. (52) to be useful, it is necessary to express the skewnesses associated with the physical variables $\varphi_{i}$ in terms of $X$ and $Y$. By definition, these satisfy

$$
\left\langle\left(\varphi_{i}-\Phi_{i}\right)\left(\varphi_{j}-\Phi_{j}\right)\left(\varphi_{k}-\Phi_{k}\right)\right\rangle \equiv \alpha_{i j k} .
$$

After substituting for the definition of these quantities inside the expectation values in Eq. (53) we arrive at the relations

$$
\begin{aligned}
& \alpha_{X X X}=\frac{1}{2 \sqrt{2}(1+\rho)^{3 / 2}}\left(\frac{\alpha_{111}}{\sigma_{1}^{3}}+3 \frac{\alpha_{112}}{\sigma_{1}^{2} \sigma_{2}}+3 \frac{\alpha_{122}}{\sigma_{1} \sigma_{2}^{2}}+\frac{\alpha_{222}}{\sigma_{2}^{3}}\right), \\
& \alpha_{X X Y}=\frac{1}{2 \sqrt{2(1-\rho)}(1+\rho)}\left(\frac{\alpha_{111}}{\sigma_{1}^{3}}+\frac{\alpha_{112}}{\sigma_{1}^{2} \sigma_{2}}-\frac{\alpha_{122}}{\sigma_{1} \sigma_{2}^{2}}-\frac{\alpha_{222}}{\sigma_{2}^{3}}\right), \\
& \alpha_{X Y Y}=\frac{1}{2 \sqrt{2(1+\rho)}(1-\rho)}\left(\frac{\alpha_{111}}{\sigma_{1}^{3}}-\frac{\alpha_{112}}{\sigma_{1}^{2} \sigma_{2}}-\frac{\alpha_{122}}{\sigma_{1} \sigma_{2}^{2}}+\frac{\alpha_{222}}{\sigma_{2}^{3}}\right), \\
& \alpha_{Y Y Y}=\frac{1}{2 \sqrt{2}(1-\rho)^{3 / 2}}\left(\frac{\alpha_{111}}{\sigma_{1}^{3}}-3 \frac{\alpha_{112}}{\sigma_{1}^{2} \sigma_{2}}+3 \frac{\alpha_{122}}{\sigma_{1} \sigma_{2}^{2}}-\frac{\alpha_{222}}{\sigma_{2}^{3}}\right) .
\end{aligned}
$$

The moments $\Phi_{i}, \Sigma_{i j}$ and $\alpha_{i j k}$ are time-dependent, but for clarity we will usually suppress this in our notation.

Next we must extract the moment hierarchy, which governs evolution of $\Phi_{i}, \sigma_{i}, \rho$ and $\alpha_{i j k}$. We expand the velocity field in a neighborhood of the instantaneous centroid $\Phi_{i}$ according to

$$
u_{i}\left(\varphi_{j}\right)=u_{i 0}+u_{i j}\left(\varphi_{j}-\Phi_{j}\right)+\frac{1}{2} u_{i j k}\left(\varphi_{j}-\Phi_{j}\right)\left(\varphi_{k}-\Phi_{k}\right)+\cdots,
$$

where we have defined

$$
\left.u_{i 0} \equiv u_{i}\right|_{\Phi_{i}},\left.\quad u_{i j} \equiv \frac{\partial u_{i}}{\partial \varphi_{j}}\right|_{\Phi_{i}}, \quad \text { and }\left.\quad u_{i j k} \equiv \frac{\partial^{2} u_{i}}{\partial \varphi_{j} \partial \varphi_{k}}\right|_{\Phi_{i}} .
$$

As in the single-field case, these coefficients are functions of time and vary with the motion of the centroid. The expansion can be pursued to higher order if desired.

Our construction of $X$ and $Y$ implies that the two-field transport equation can be arranged as a double Gauss-Hermite expansion,

$$
\frac{\partial P\left(\varphi_{i}, N\right)}{\partial N}+\frac{\partial}{\partial \varphi_{i}}\left[u_{i} P\left(\varphi_{i}, N\right)\right]=P_{g} \sum_{m, n \geq 0} c_{m n} H_{m}(X) H_{n}(Y)=0 .
$$

Because the Hermite polynomials are orthogonal in the measure defined by $P_{g}$, we deduce the moment hierarchy

$$
c_{m n}=0 .
$$


We define the "rank" $r$ of each coefficient $c_{m n}$ by $r \equiv m+n$. We terminated the velocity field expansion at quadratic order, and our probability distribution included only the first three moments. It follows that only $c_{m n}$ with rank five or less are nonzero. If we followed the velocity field to higher order, or included higher terms in the moment expansion, we would obtain non-trivial higher-rank coefficients. Inclusion of additional coefficients requires no qualitative modification of our analysis and can be incorporated in the scheme we describe below.

A useful feature of the expansion in Eq. (61) is that the rank- $r$ coefficients give evolution equations for the order- $r$ moments. Written explicitly in components, the expressions that result from (61) are quite cumbersome. However, when written as field-space covariant expressions they can be expressed in a surprisingly compact form.

Rank 0 The rank-0 coefficient $c_{00}$ is identically zero. This expresses the fact that the total probability is conserved as the distribution evolves.

Rank 1 The rank-1 coefficients $c_{01}$ and $c_{10}$ give evolution equations for the centroid $\Phi_{i}$. These equations can be written in the form

$$
\frac{\mathrm{d} \Phi_{i}}{\mathrm{~d} N}=u_{i 0}+\frac{1}{2} \Sigma_{j k} u_{i j k}
$$

We remind the reader that here and below, terms like $u_{i 0}, u_{i j}$ and $u_{i j k}$ represent the velocity field and its derivatives evaluated at the centroid $\Phi_{i}$. The first term in (63) expresses the non-anomalous motion of the centroid, which coincides with the background velocity field of Eq. (43). The second term describes how the wings of the probability distribution sample the velocity field at nearby points. Narrow probability distributions have small components of $\Sigma$ and hence are only sensitive to the local value of $u_{i}\left(\varphi_{j}\right)$. Broad probability distributions have large components of $\Sigma$ and are therefore more sensitive to the velocity field far from the centroid.

Rank 2 The rank-2 coefficients $c_{02}, c_{11}$ and $c_{20}$ give evolution equations for the variances $\sigma_{i}^{2}$ and the correlation $\rho$. These can conveniently be packaged as evolution equations for the matrix $\Sigma$

$$
\frac{\mathrm{d} \Sigma_{i j}}{\mathrm{~d} N}=u_{i k} \Sigma_{k j}+u_{j k} \Sigma_{k i}+\frac{1}{2}\left(\alpha_{i m n} u_{j m n}+\alpha_{j m n} u_{i m n}\right)
$$

This equation describes the stretching and rotation of $\Sigma$ as it is transported by the velocity field. It includes a sensitivity to the wings of the probability distribution, in a manner analogous to the similar term appearing in (41). Hence the skew $\alpha_{i j k}$ acts as a source for the correlation matrix.

Rank 3 The rank-3 coefficients $c_{03}, c_{12}, c_{21}$ and $c_{30}$ describe evolution of the moments $\alpha_{i j k}$. These are

$$
\begin{aligned}
\frac{\mathrm{d} \alpha_{i j k}}{\mathrm{~d} N}=u_{i n} \alpha_{n j k} & +\Sigma_{j m} u_{i m n} \Sigma_{n k} \\
& + \text { cyclic permutations } i \rightarrow j \rightarrow k .
\end{aligned}
$$

The first term describes how the moments flow into each other as the velocity field rotates and shears the $(X, Y)$ coordinate frame relative to the $\varphi_{i}$ coordinate frame. 
The second term describes sourcing of non-Gaussianity from inhomogeneities in the velocity field and the overall spread of the probability distribution.

Some higher-rank coefficients - in our case, those of ranks four and five - are also nonzero, but do not give any new evolution equations. These coefficients measure the "error" introduced by truncating the moment expansion. If we had included higher cumulants, these higher-rank coefficients would have given evolution equations for the higher moments of the probability distribution. In general, all moments of the density function will mix so it is always necessary to terminate our expansion at a predetermined order-both in cumulants and powers of the field fluctuation. The order we have chosen is sufficient to generate evolution equations containing both the leading-order behavior of the moments - namely, the first terms in Eqs. (63), (64) and (65) - and the leading corrections, given by the latter terms in these equations.

\section{Numerical results}

At this point we put our new method into practice. We study two models for which the non-Gaussian signal is already known, using the standard $\delta N$ formula. For each case we employ our method and compare it with results obtained using $\delta N$. To ensure a fair comparison, we solve numerically in both cases. Our new method employs the slow-roll approximation, as described above. Therefore, when using the $\delta N$ approach we produce results both with and without slow-roll simplifications.

First consider double quadratic inflation, which was studied by Rigopoulos, Shellard \& van Tent [36, 63] and later by Vernizzi \& Wands [19]. The potential is

$$
V(\phi, \chi)=\frac{1}{2} m_{\phi}^{2} \phi^{2}+\frac{1}{2} m_{\chi}^{2} \chi^{2} .
$$

We use the initial conditions chosen in Ref. [36], where $m_{\phi} / m_{\chi}=9$, and the fiducial trajectory has coordinates $\phi^{\star}=8.2$ and $\chi^{\star}=12.9$.

We plot the evolution of $f_{\mathrm{NL}}$ in Fig. 1, which also shows the prediction of the standard $\delta N$ formula (with and without employing slow roll simplifications). We implement the $\delta N$ algorithm using a finite difference method to calculate the derivatives of $N$. A similar technique was used in Ref. [19]. This model yields a very modest nonGaussian signal, below unity even at its peak. If inflation ends away from the spike then $f_{\mathrm{NL}}$ is practically negligible.

Eq. (12) shows that the method of moment transport allows us to separate contributions to $f_{\mathrm{NL}}$ from the intrinsic non-Gaussianity of the field fluctuations, and non-linearities of the gauge transformation to $\zeta$. As explained in $\$ 2.2$, we denote the former $f_{\mathrm{NL} 1}$ and the latter $f_{\mathrm{NL} 2}$, and plot them separately in Fig. 2, Inspection of this figure clearly shows that $f_{\mathrm{NL}}$ is determined by a cancellation between two much larger components. Its final shape and magnitude are exquisitely sensitive to their relative phase. Initially, the magnitudes of $f_{\mathrm{NL} 1}$ and $f_{\mathrm{NL} 2}$ grow, but their sum remains small. The peak in Fig. 1 arises from the peak of $f_{\mathrm{NL} 2}$, which is incompletely cancelled 


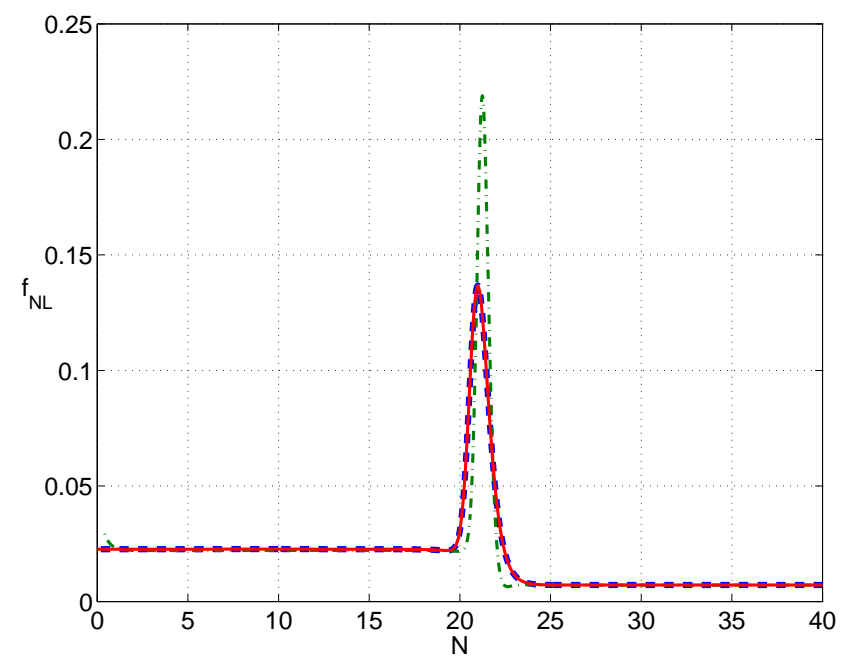

Figure 1. Evolution of $f_{\mathrm{NL}}$ in double quadratic inflation. The solid red line is obtained by numerically solving the moment transport equations obtained in 33 , The blue dashed line and green dot-dashed line are the output of a numerical implementation of the standard $\delta N$ approach, with and without slow roll respectively, using the fiducial picture. The red and blue lines lie on top of each other.

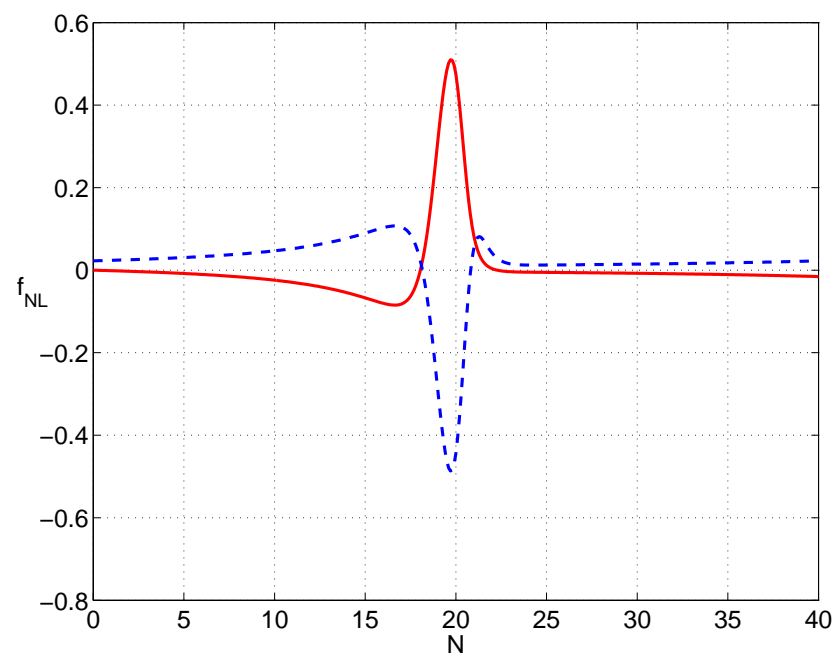

Figure 2. Evolution of $f_{\mathrm{NL} 1}$ (solid red line), measuring the contribution of intrinsic non-linearities among the field fluctuations; and $f_{\mathrm{NL} 2}$ (dashed blue line), measuring the contribution of the gauge transformation to $\zeta$. 


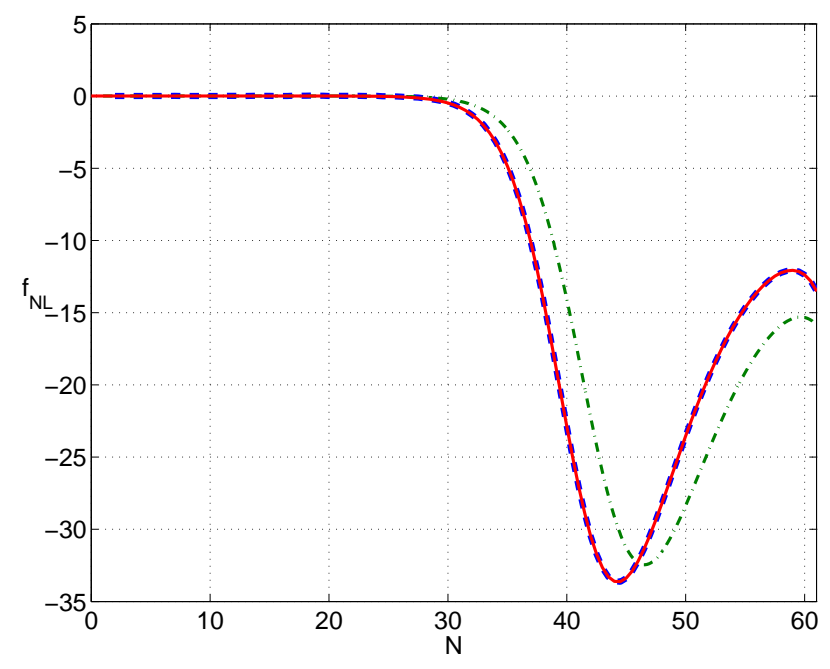

Figure 3. Evolution of $f_{\mathrm{NL}}$, for the potential $V=V_{0} \chi^{2} e^{-\lambda \phi^{2}}$ (Example A from $\S 5$ of Ref. [21]). The solid red line represents the method of moment transport, whereas the blue dashed line and green dot-dashed line represents the output of conventional numerical $\delta N$ with and without slow-roll respectively. The red and blue lines are again coincident.

by $f_{\mathrm{NL1}}$. It is remarkable that $f_{\mathrm{NL1}}$ initially evolves in exact opposition to the gauge transformation, to which it is not obviously connected.

In the double quadratic model, $f_{\mathrm{NL}}$ is always small. However, it has recently been shown by Byrnes et al. that a large non-Gaussian signal can be generated even when slow-roll is a good approximation [21, 22]. The conditions for this to occur are incompletely understood, but apparently require a specific choice of potential and strong tuning of initial conditions. In Figs. 3 4 we show the evolution of $f_{\mathrm{NL}}$ in a model with the potential

$$
V=V_{0} \chi^{2} e^{-\lambda \phi^{2}}
$$

which corresponds to Example A of Ref. [21, §5] when we choose $\lambda=0.05$ and initial conditions $\chi^{\star}=16, \phi^{\star}=0.001$. It is clear that the agreement is exact. In this model, $f_{\mathrm{NL}}$ is overwhelmingly dominated by the contribution from the second-order gauge transformation, $f_{\mathrm{NL} 2}$, as shown in Fig. 4. This conclusion applies equally to the other large- $f_{\mathrm{NL}}$ examples discussed in Refs. [21, 22], although we make no claim that this is a general phenomenon.

In conclusion, Figs. 1 and 3 show excellent agreement between our new method and the outcome of the numerical $\delta N$ formula. These figures also compare the moment transport method and $\delta N$ without the slow-roll approximation. We conclude that the slow-roll estimate remains broadly accurate throughout the entire evolution. 


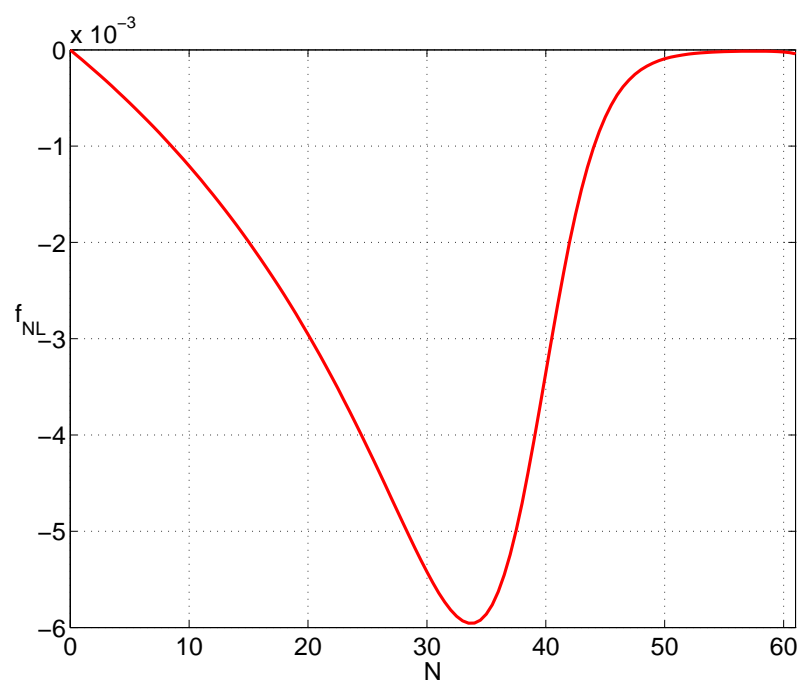

Figure 4. Evolution of $f_{\mathrm{NL} 1}$, for the potential $V=V_{0} \chi^{2} e^{\lambda \phi^{2}}$. Comparison with Fig. 3 shows that $f_{\mathrm{NL}}$ is totally dominated by $f_{\mathrm{NL} 2}$.

\section{Discussion}

Non-linearities are now routinely extracted from all-sky observations of the microwave background anisotropy. Our purpose in this paper has been to propose a new technique with which to predict the observable signal. Present data already give interesting constraints on the skewness parameter $f_{\mathrm{NL}}$, and over the next several years we expect that the Planck survey satellite will make these constraints very stringent. It is even possible that higher-order moments, such as the kurtosis parameter $g_{\mathrm{NL}}[64$ ] will become better constrained [65]. To meet the need of the observational community for comparison with theory, reliable estimates of these non-linear quantities will be necessary for various models of early-universe physics.

A survey of the literature suggests that the 'conventional' $\delta N$ method, originally introduced by Lyth \& Rodríguez, remains the method of choice for analytical study of non-Gaussianity. In comparison, our proposed moment transport method exhibits several clear differences. First, the conventional method functions best when we base the $\delta N$ expansion on a flat hypersurface immediately after horizon exit. In our method, we make the opposite choice and move the flat hypersurface as close as possible to the time of observation. After this, the role of the $\delta N$ formula is to provide no more than the non-linear gauge transformation between field fluctuations and the curvature perturbation. We substitute the method of moment transport to evolve the distribution of field fluctuations between horizon exit and observation.

Second, in integrating the transport equation one uses an expansion of the velocity field such as the one given in Eqs. (59)-(60). This expansion is refreshed at each step of integration, so the result is related to conventional perturbative calculations in a very similar way to renormalization-group improved perturbation theory [66]. In this 
interpretation, derivatives of $u_{i}$ play the role of couplings. At a given order, $m$, in the moment hierarchy, the equations for lower-order moments function as renormalization group equations for the couplings at level- $m$, resumming potentially large terms before they spoil perturbation theory. This property is shared with any formalism such as $\delta N$ which is non-perturbative in time evolution, but may be an advantage in comparison with perturbative methods. We also note that although $\delta N$ is non-perturbative as a point of principle, practical implementations are frequently perturbative. For example, the method of Vernizzi \& Wands [19] and Battefeld \& Easther [20] depends on the existence of quantities which are conserved only to leading order in $\epsilon N$, and can lose accuracy after $N \sim \epsilon^{-1}$ e-foldings.

Numerical calculations confirm that our method gives results in excellent agreement with existing techniques. As a by-product of our analysis, we note that the large non-gaussianities which have recently been observed in sum- and product-separable potentials [21, 22] are dominated by non-linearities from the second-order part of the gauge transformation from $\delta \varphi_{i}$ to $\zeta$. The contribution from intrinsic non-linearities of the field fluctuations, measured by the skewnesses $\alpha_{i j k}$, is negligible. In such cases one can obtain a useful formula for $f_{\mathrm{NL}}$ by approximating the field distribution as an exact Gaussian. The non-Gaussianity produced in such cases arises from a distortion of comoving hypersurfaces with respect to adjacent spatially flat hypersurfaces.

Our new method joins many well-established techniques for estimating nonGaussian properties of the curvature perturbation. In our experience, these techniques give comparable estimates of $f_{\mathrm{NL}}$, but they do not exactly agree. Each method invokes different assumptions, such as the neglect of gradients or the degree to which time dependence can be accommodated. The mutual scatter between different methods can be attributed to the theory error inherent in any estimate of $f_{\mathrm{NL}}$. The comparison presented in $\$ 4$ shows that while all of these methods slightly disagree, the moment transport method gives good agreement with other established methods.

\section{Acknowledgments}

DM is supported by the Cambridge Centre for Theoretical Cosmology (CTC). DS is funded by STFC. DW acknowledges support from the CTC. We would like to thank Chris Byrnes, Jim Lidsey and Karim Malik for helpful conversations.

\section{References}

[1] S. W. Hawking, The Development of Irregularities in a Single Bubble Inflationary Universe, Phys. Lett. B115 (1982) 295.

[2] S. W. Hawking and I. G. Moss, Fluctuations in the inflationary universe, Nucl. Phys. B224 (1983) 180.

[3] J. M. Bardeen, P. J. Steinhardt, and M. S. Turner, Spontaneous Creation of Almost Scale - Free Density Perturbations in an Inflationary Universe, Phys. Rev. D28 (1983) 679.

[4] D. H. Lyth, Large Scale Energy Density Perturbations and Inflation, Phys. Rev. D31 (1985) 1792-1798. 
[5] A. H. Guth and S.-Y. Pi, The Quantum Mechanics of the Scalar Field in the New Inflationary Universe, Phys. Rev. D32 (1985) 1899-1920.

[6] T. Falk, R. Rangarajan, and M. Srednicki, The angular dependence of the three-point correlation function of the cosmic microwave background radiation as predicted by inflationary cosmologies, Astrophys. J. 403 (1993) L1, arXiv:astro-ph/9208001.

[7] A. Gangui, F. Lucchin, S. Matarrese, and S. Mollerach, The three-point correlation function of the cosmic microwave background in inflationary models, Astrophys. J. 430 (1994) 447-457, arXiv:astro-ph/9312033.

[8] T. Pyne and S. M. Carroll, Higher-Order Gravitational Perturbations of the Cosmic Microwave Background, Phys. Rev. D53 (1996) 2920-2929, arXiv:astro-ph/9510041].

[9] V. Acquaviva, N. Bartolo, S. Matarrese, and A. Riotto, Second-order cosmological perturbations from inflation, Nucl. Phys. B667 (2003) 119-148, arXiv:astro-ph/0209156.

[10] J. M. Maldacena, Non-Gaussian features of primordial fluctuations in single field inflationary models, JHEP 05 (2003) 013, arXiv:astro-ph/0210603.

[11] D. H. Lyth and Y. Rodriguez, The inflationary prediction for primordial non-gaussianity, Phys. Rev. Lett. 95 (2005) 121302, arXiv:astro-ph/0504045.

[12] D. Seery and J. E. Lidsey, Primordial non-gaussianities from multiple-field inflation, JCAP 0509 (2005) 011, arXiv:astro-ph/0506056.

[13] E. Komatsu et al., Non-Gaussianity as a Probe of the Physics of the Primordial Universe and the Astrophysics of the Low Redshift Universe, arXiv:0902.4759.

[14] E. Komatsu and D. N. Spergel, Acoustic signatures in the primary microwave background bispectrum, Phys. Rev. D63 (2001) 063002, arXiv:astro-ph/0005036].

[15] R. Juszkiewicz, D. H. Weinberg, P. Amsterdamski, M. Chodorowski, and F. Bouchet, Weakly nonlinear Gaussian fluctuations and the Edgeworth expansion, Astrophys. J. 442 (1995) 39.

[16] F. R. Bouchet and R. Juszkiewicz, Perturbation theory confronts observations: Implications for the 'initial' conditions and $\Omega$, arXiv:astro-ph/9312007.

[17] F. R. Bouchet, Introductory overview of Eulerian and Lagrangian perturbation theories, arXiv:astro-ph/9603013.

[18] P. Fosalba, E. Gaztanaga, and E. Elizalde, Gravitational Evolution of the Large-Scale Density Distribution: The Edgeworth \& Gamma Expansions, arXiv:astro-ph/9910308.

[19] F. Vernizzi and D. Wands, Non-Gaussianities in two-field inflation, JCAP 0605 (2006) 019, arXiv:astro-ph/0603799.

[20] T. Battefeld and R. Easther, Non-gaussianities in multi-field inflation, JCAP 0703 (2007) 020, arXiv:astro-ph/0610296.

[21] C. T. Byrnes, K.-Y. Choi, and L. M. H. Hall, Conditions for large non-Gaussianity in two-field slow-roll inflation, JCAP 0810 (2008) 008, arXiv:0807.1101.

[22] C. T. Byrnes, K.-Y. Choi, and L. M. H. Hall, Large non-Gaussianity from two-component hybrid inflation, JCAP 0902 (2009) 017, arXiv:0812.0807.

[23] C. T. Byrnes and G. Tasinato, Non-Gaussianity beyond slow roll in multi-field inflation, arXiv:0906.0767.

[24] D. Battefeld and T. Battefeld, On Non-Gaussianities in Multi-Field Inflation (N fields): Bi- and Tri-spectra beyond Slow-Roll, arXiv:0908.4269.

[25] D. Seery and J. E. Lidsey, Non-gaussianity from the inflationary trispectrum, JCAP 0701 (2007) 008, arXiv:astro-ph/0611034].

[26] C. T. Byrnes, M. Sasaki, and D. Wands, The primordial trispectrum from inflation, Phys. Rev. D74 (2006) 123519, arXiv:astro-ph/0611075].

[27] S. Dimopoulos, S. Kachru, J. McGreevy, and J. G. Wacker, N-flation, JCAP 0808 (2008) 003, arXiv:hep-th/0507205.

[28] J.-L. Lehners and S. Renaux-Petel, Multifield Cosmological Perturbations at Third Order and the Ekpyrotic Trispectrum, arXiv:0906.0530.

[29] P. Creminelli, On non-gaussianities in single-field inflation, JCAP 0310 (2003) 003, 
arXiv:astro-ph/0306122.

[30] M. Zaldarriaga, Non-Gaussianities in models with a varying inflaton decay rate, Phys. Rev. D69 (2004) 043508, arXiv:astro-ph/0306006.

[31] N. Arkani-Hamed, P. Creminelli, S. Mukohyama, and M. Zaldarriaga, Ghost Inflation, JCAP 0404 (2004) 001, arXiv:hep-th/0312100.

[32] M. Alishahiha, E. Silverstein, and D. Tong, DBI in the sky, Phys. Rev. D70 (2004) 123505, arXiv:hep-th/0404084.

[33] A. A. Starobinsky, Multicomponent de Sitter (Inflationary) Stages and the Generation of Perturbations, JETP Lett. 42 (1985) 152-155.

[34] M. Sasaki and E. D. Stewart, A General analytic formula for the spectral index of the density perturbations produced during inflation, Prog. Theor. Phys. 95 (1996) 71-78, arXiv:astro-ph/9507001.

[35] G. I. Rigopoulos and E. P. S. Shellard, Non-linear inflationary perturbations, JCAP 0510 (2005) 006, arXiv:astro-ph/0405185].

[36] G. I. Rigopoulos, E. P. S. Shellard, and B. J. W. van Tent, Non-linear perturbations in multiple-field inflation, Phys. Rev. D73 (2006) 083521, arXiv:astro-ph/0504508.

[37] D. Langlois and F. Vernizzi, Evolution of non-linear cosmological perturbations, Phys. Rev. Lett. 95 (2005) 091303, arXiv:astro-ph/0503416.

[38] D. Langlois and F. Vernizzi, Conserved non-linear quantities in cosmology, Phys. Rev. D72 (2005) 103501, arXiv:astro-ph/0509078.

[39] D. Langlois and F. Vernizzi, Nonlinear perturbations of cosmological scalar fields, JCAP 0702 (2007) 017, arXiv:astro-ph/0610064.

[40] I. Huston and K. A. Malik, Numerical calculation of second order perturbations, arXiv:0907.2917.

[41] D. H. Lyth, The curvature perturbation in a box, JCAP 0712 (2007) 016, arXiv:0707.0361.

[42] M. S. Sloth, On the one loop corrections to inflation and the CMB anisotropies, Nucl. Phys. B748 (2006) 149-169, arXiv:astro-ph/0604488].

[43] M. S. Sloth, On the one loop corrections to inflation. II: The consistency relation, Nucl. Phys. B775 (2007) 78-94, arXiv:hep-th/0612138.

[44] D. Seery, One-loop corrections to the curvature perturbation from inflation, JCAP 0802 (2008) 006, arXiv:0707.3378.

[45] H. R. S. Cogollo, Y. Rodríguez, and C. A. Valenzuela-Toledo, On the Issue of the $\zeta$ Series Convergence and Loop Corrections in the Generation of Observable Primordial Non-Gaussianity in Slow-Roll Inflation. Part I: the Bispectrum, JCAP 0808 (2008) 029, arXiv:0806.1546.

[46] Y. Rodríguez and C. A. Valenzuela-Toledo, On the Issue of the $\zeta$ Series Convergence and Loop Corrections in the Generation of Observable Primordial Non-Gaussianity in Slow-Roll Inflation. Part II: the Trispectrum, arXiv:0811.4092.

[47] D. Seery, M. S. Sloth, and F. Vernizzi, Inflationary trispectrum from graviton exchange, JCAP 0903 (2009) 018, arXiv:0811.3934.

[48] D. Seery, K. A. Malik, and D. H. Lyth, Non-gaussianity of inflationary field perturbations from the field equation, JCAP 0803 (2008) 014, arXiv:0802.0588.

[49] K.-Y. Choi, L. M. H. Hall, and C. van de Bruck, Spectral running and non-Gaussianity from slow-roll inflation in generalised two-field models, JCAP 0702 (2007) 029, arXiv:astro-ph/0701247.

[50] S. Yokoyama, T. Suyama, and T. Tanaka, Primordial Non-Gaussianity in Multi-Scalar Inflation, Phys. Rev. D77 (2008) 083511, arXiv:0711.2920.

[51] J. Garcia-Bellido and D. Wands, Metric perturbations in two-field inflation, Phys. Rev. D53 (1996) 5437-5445, arXiv:astro-ph/9511029.

[52] C. Gordon, D. Wands, B. A. Bassett, and R. Maartens, Adiabatic and entropy perturbations from inflation, Phys. Rev. D63 (2001) 023506, arXiv:astro-ph/0009131.

[53] A. Taylor and P. Watts, Evolution of the cosmological density distribution function, 
arXiv:astro-ph/0001118.

[54] L. Amendola, Non Gaussian likelihood function and COBE data, Mon. Not. Roy. Astron. Soc. 283 (1996) 983-989.

[55] S. Matarrese, L. Verde, and R. Jimenez, The abundance of high-redshift objects as a probe of nonGaussian initial conditions, Astrophys. J. 541 (2000) 10, arXiv:astro-ph/0001366.

[56] L. Amendola, The dependence of cosmological parameters estimated from the microwave background on non-gaussianity, Astrophys. J. 569 (2002) 595-599, arXiv:astro-ph/0107527.

[57] P. Watts and P. Coles, Statistical Cosmology with Quadratic Density Fields, Mon. Not. Roy. Astron. Soc. 338 (2003) 806, arXiv:astro-ph/0208295].

[58] M. LoVerde, A. Miller, S. Shandera, and L. Verde, Effects of Scale-Dependent Non-Gaussianity on Cosmological Structures, JCAP 0804 (2008) 014, arXiv:0711.4126.

[59] D. Seery and J. C. Hidalgo, Non-Gaussian corrections to the probability distribution of the curvature perturbation from inflation, JCAP 0607 (2006) 008, arXiv:astro-ph/0604579.

[60] T. Y. Lam and R. K. Sheth, Halo abundances in the $f_{\mathrm{NL}}$ model, arXiv:0905.1702.

[61] S. Blinnikov and R. Moessner, Expansions for nearly Gaussian distributions, Astron. Astrophys. Suppl. Ser. 130 (1998) 193-205, arXiv:astro-ph/9711239.

[62] A. A. Starobinsky, Stochastic de Sitter (inflationary) stage in the early universe, . In De Vega, H.J. (Ed.), Sanchez, N. (Ed.): Field Theory, Quantum Gravity and Strings, 107-126.

[63] G. I. Rigopoulos, E. P. S. Shellard, and B. J. W. van Tent, Quantitative bispectra from multifield inflation, Phys. Rev. D76 (2007) 083512, arXiv:astro-ph/0511041.

[64] M. Sasaki, J. Valiviita, and D. Wands, Non-gaussianity of the primordial perturbation in the curvaton model, Phys. Rev. D74 (2006) 103003, arXiv:astro-ph/0607627.

[65] V. Desjacques and U. Seljak, Signature of primordial non-Gaussianity of $\phi^{3}$-type in the mass function and bias of dark matter haloes, arXiv:0907.2257.

[66] M. Gell-Mann and F. E. Low, Quantum electrodynamics at small distances, Phys. Rev. 95 (1954) 1300-1312. 